\title{
Lightcurves of 10 Hygiea, 241 Germania and 509 Iolanda
}

\author{
M.J. López-González and E. Rodríguez \\ Instituto de Astrofísica de Andalucía, CSIC, P.O. Box 3004, E-18080 Granada, Spain \\ Received February 23; accepted May 10, 2000
}

\begin{abstract}
The asteroids 10 Hygiea, 241 Germania and 509 Iolanda have been studied photometrically. From their lightcurves, synodic periods of $27.63 \pm 0.02,15.51 \pm 0.01$ and $12.72 \pm 0.02$ hours, and amplitudes of $0.28,0.17$ and 0.45 , have been deduced for 10 Hygiea, 241 Germania and 509 Iolanda, respectively. The period found for 241 Germania is a significant revision from one previously published, and we present a first indication of pole orientation and shape. A synodic period is obtained for 509 Iolanda. The solution obtained for the sense of rotation, sidereal period, pole orientation and shape properties for 10 Hygiea confirms previous results.
\end{abstract}

Key words: minor planets, asteroids - techniques: photometric

\section{Introduction}

Lightcurves from different aspects, as an asteroid orbits, provide information about spin period, axis orientation and body shape. 10 Hygiea is a well observed asteroid. It has been observed in eight oppositions. However, 241 Germania has been observed in only two oppositions and there was no previous lightcurve available for 509 Iolanda.

In this paper we present lightcurves of 10 Hygiea, 241 Germania and 509 Iolanda taken during SeptemberOctober 1996 from the observatory of Sierra Nevada, Spain. Simultaneous uvby photometry was obtained and used to derive Strömgren and Johnson reduced magnitudes and colour indices. Rotational and shape parameters are obtained for 10 Hygiea that confirm previous results. New indications about the pole orientation and the shape parameters for 241 Germania are presented and a synodic period is estimated for 509 Iolanda.

Send offprint requests to: M.J. López-González, e-mail: mariajose@iaa.es

\section{Observations}

The observations were carried out on different nights of September-October 1996 using the $90 \mathrm{~cm}$ telescope at Sierra Nevada Observatory, Spain. This telescope is equipped with a six channel uvby $\beta$ photometer for simultaneous measurements in uvby or in the $\mathrm{H}_{\beta}$ channels respectively (Nielsen 1983), but only uvby measurements were collected during this observing run.

In order to make differential photometry, the comparison stars were chosen taking into account their spectral type (close to solar) and in the neighbourhood of the asteroids for better reduction of the data avoiding extinction problems. $\mathrm{C} 1=\mathrm{SAO} 92273\left(V=8^{\mathrm{m}} 5, \mathrm{~K} 0\right)$ was used as the main comparison star and $\mathrm{C} 2=\mathrm{SAO} 92119(V=10 \mathrm{~m} 0$, G0), C3=SAO $92152\left(V=9 \mathrm{~m}^{\mathrm{m}} 4, \mathrm{G} 5\right)$ and $\mathrm{C} 4=\mathrm{SAO} 74083$ $(V=6.7$, A2) as check stars. During the observations reported here, neither of the comparison stars showed any sign of variability within 0.005 .

To transform our data into the standard uvby system, we have used the same procedure described in Rodríguez et al. (1997). After differential magnitudes in the standard system were obtained for the asteroids with respect to the main comparison star $(\mathrm{C} 1=\mathrm{SAO} 92273)$, we correct them to unit distance from the sun and the earth and perform light-time corrections to all the observations. Then, we transform these differential magnitudes to absolute magnitudes using the standard values of $\mathrm{C} 4, V=6.70$, $b-y=0{ }^{\mathrm{m}} 046, m_{1}=00^{\mathrm{m}} 161$ and $c_{1}=1.193$, listed in the Hauck \& Mermilliod (1998) catalogue. In addition, we obtained the following values of $V=8^{\mathrm{m}} 52,10^{\mathrm{m}} 08,9^{\mathrm{m}} \cdot 33$ for $\mathrm{C} 1, \mathrm{C} 2$ and $\mathrm{C} 3$, respectively.

\section{Analysis}

In Table 1 the aspect data for each asteroid, the longitude and latitude relative to the ecliptic, the solar phase angle, the geocentric, $r$, and heliocentric, $\Delta$, distances, are listed for every day of observation. 
Table 1. Aspect data

\begin{tabular}{lccccc}
\hline $\begin{array}{l}\text { Date } \\
\text { (0 UT) }\end{array}$ & $\begin{array}{c}\text { Long } \\
(1950)\end{array}$ & $\begin{array}{c}\text { Lat } \\
(1950)\end{array}$ & $\begin{array}{c}\text { Phase } \\
(\mathrm{deg})\end{array}$ & $\begin{array}{c}r \\
(\mathrm{AU})\end{array}$ & $\begin{array}{c}\Delta \\
(\mathrm{AU})\end{array}$ \\
\hline 10 Hygiea & & & & & \\
22 09 1996 & 20.85 & 5.34 & 6.60 & 2.43850 & 3.38599 \\
04 10 1996 & 18.63 & 5.42 & 2.89 & 2.40571 & 3.39552 \\
07 10 1996 & 18.04 & 5.43 & 2.10 & 2.40397 & 3.39785 \\
08 10 1996 & 17.84 & 5.43 & 1.89 & 2.40398 & 3.39862 \\
241 Germania & & & & & \\
04 10 1996 & 18.94 & 8.25 & 4.23 & 1.80108 & 2.78756 \\
07 10 1996 & 18.32 & 8.24 & 3.43 & 1.79872 & 2.78908 \\
08 10 1996 & 18.11 & 8.23 & 3.23 & 1.79848 & 2.78959 \\
509 Iolanda & & & & & \\
04 10 1996 & 21.52 & 8.22 & 4.90 & 1.81454 & 2.79611 \\
07 10 1996 & 20.88 & 7.99 & 3.90 & 1.80876 & 2.79640 \\
08 10 1996 & 20.66 & 7.91 & 3.60 & 1.80740 & 2.79650 \\
\hline
\end{tabular}

\subsection{Photometry}

These observations were carried out during three nights of October 1996 when the asteroids were very close to the opposition. 10 Hygiea was observed one additional night of September when the solar phase angle of this asteroid was $4^{\circ}$ greater than its average phase angle corresponding to October observations. The absolute magnitudes obtained during September for 10 Hygiea are about 0. 28 greater than those obtained during October. In Table 2 the mean reduced magnitudes observed for each uvby Strömgren filter, $\overline{u v b y}(1, \alpha)$, the observed Strömgren colour indices, $b-y$ and $u-b$, and the average phase angle of October observations, $\bar{\alpha}$, for each asteroid, are presented.

These Strömgren values are transformed into the $U B V$ Johnson system by using the equations of Warren \& Hesser (1977) to transform the Strömgren $b-y$ and $u-b$ colour indices to Johnson $B-V$ and $U-B$ colour indices. The Johnson magnitudes and colour indices obtained, $\overline{U B V}(1, \alpha)$, are also shown in Table 2.

Rotational synodic periods for each asteroid have been obtained performing analysis of frequencies on our data using the method described in Rodríguez et al. (1998). Synodic periods of $1.1511,0.6464$ and 0.5300 , and estimated errors, after optimizing these periods using a nonlinear least squares fit, of $0.0007,0.0006$ and 0.0010 are obtained for 10 Hygiea, 241 Germania and 509 Iolanda, respectively. Then, synodic periods of $27.63 \pm 0.02$ hours for 10 Hygiea, $15.51 \pm 0.01$ hours for 241 Germania and $12.72 \pm 0.02$ hours for 509 Iolanda are derived from these observations.

Figures 1, 3, and 6 show the composite lightcurves derived using these synodic periods for each asteroid. The uvby $(1, \alpha)$ magnitudes and the $b-y, v-b$ and $u-b$ colour indices versus the rotational phase are shown in these figures. The lightcurves obtained for each asteroid show regular shapes with two maxima and two minima per rotational cycle. We have not applied any phase correction to these magnitudes, however the magnitudes measured for

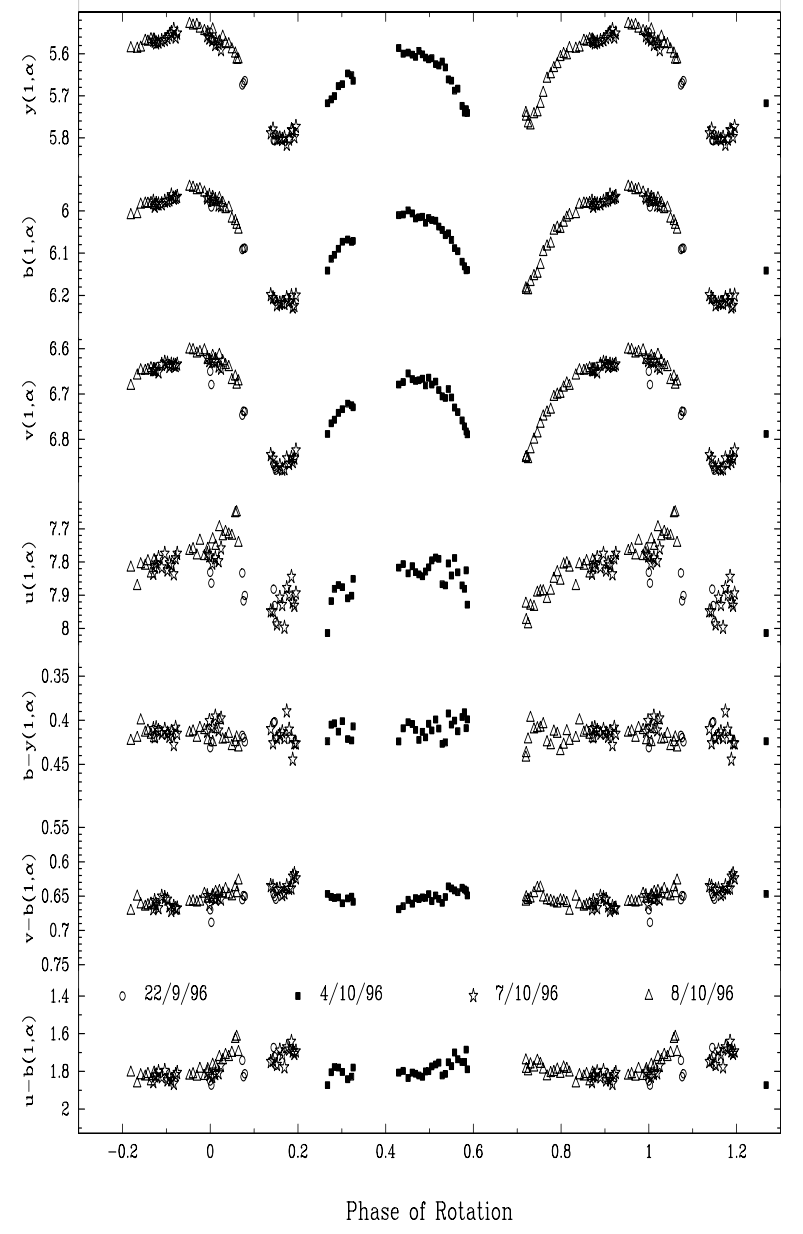

Fig. 1. Lightcurves and colour indices of 10 Hygiea in rotational phase. The 0 phase time corresponds to JD 2450349.4941 corrected for light-time

10 Hygiea on September 22, when the solar phase angle was greater, have been shifted by an additional constant of -0.28 in all the uvby filters.

\subsection{Poles and shapes}

Poles and shapes for these asteroids have been determined using the Epoch/Amplitude method (see Taylor 1979; Magnusson 1986; Magnusson et al. 1989; Michalowski \& Velichko 1990; De Angelis 1993). We used the method to resolve the Epoch and Amplitude equations as it was explained in López-González \& Rodríguez (1999). In this way we obtain the most likely solution for the sidereal period of rotation of the asteroid, $\mathrm{Tsid}$, the pole coordinates of the asteroid, $\lambda_{\mathrm{p}}$ and $\beta_{\mathrm{p}}$, the ratios of the symmetry axes of the asteroid (considered as a triaxial ellipsoid of axis $a \geq b \geq c$ rotating about their shortest axis), $a / b$ and 
Table 2. Photometry

\begin{tabular}{rrrrrr}
\hline $\begin{array}{c}\text { uvby }(1, \alpha) \\
(\mathrm{mag})\end{array}$ & $\begin{array}{c}\overline{U B V}(1, \alpha) \\
(\mathrm{mag})\end{array}$ & $\begin{array}{c}\overline{u v b y}(1, \alpha) \\
(\mathrm{mag})\end{array}$ & $\begin{array}{c}\overline{U B V}(1, \alpha) \\
(\mathrm{mag})\end{array}$ & $\begin{array}{c}\overline{u v b y}(1, \alpha) \\
(\mathrm{mag})\end{array}$ & $\begin{array}{c}\overline{U B V}(1, \alpha) \\
(\mathrm{mag})\end{array}$ \\
\hline 10 Hygiea & $(\bar{\alpha}=2.2 \pm 0.5)$ & 241 Germania & $(\bar{\alpha}=3.5 \pm 0.5)$ & 509 Iolanda & $(\bar{\alpha}=4.0 \pm 0.7)$ \\
$y 5.64 \pm 0.08$ & $V 5.64 \pm 0.08$ & $y 7.98 \pm 0.05$ & $V 7.98 \pm 0.05$ & $y 9.04 \pm 0.13$ & $V 9.04 \pm 0.13$ \\
$b 6.05 \pm 0.08$ & $B 6.30 \pm 0.10$ & $b 8.38 \pm 0.06$ & $B 8.62 \pm 0.09$ & $b 9.48 \pm 0.12$ & $B 9.74 \pm 0.24$ \\
$v 6.71 \pm 0.08$ & $U 6.61 \pm 0.15$ & $v 9.03 \pm 0.06$ & $U 8.88 \pm 0.13$ & $v 10.22 \pm 0.15$ & $U 10.17 \pm 0.46$ \\
$u$ 7.84 0.07 & & $u 10.09 \pm 0.09$ & & $u 11.42 \pm 0.30$ & \\
$b-y 0.41 \pm 0.01$ & $B-V 0.66 \pm 0.02$ & $b-y 0.40 \pm 0.02$ & $B-V 0.64 \pm 0.04$ & $b-y 0.44 \pm 0.07$ & $B-V 0.70 \pm 0.12$ \\
$u-b 1.78 \pm 0.05$ & $U-B 0.31 \pm 0.04$ & $u-b 1.71 \pm 0.06$ & $U-B 0.26 \pm 0.04$ & $u-b 1.94 \pm 0.30$ & $U-B 0.43 \pm 0.22$ \\
\hline
\end{tabular}

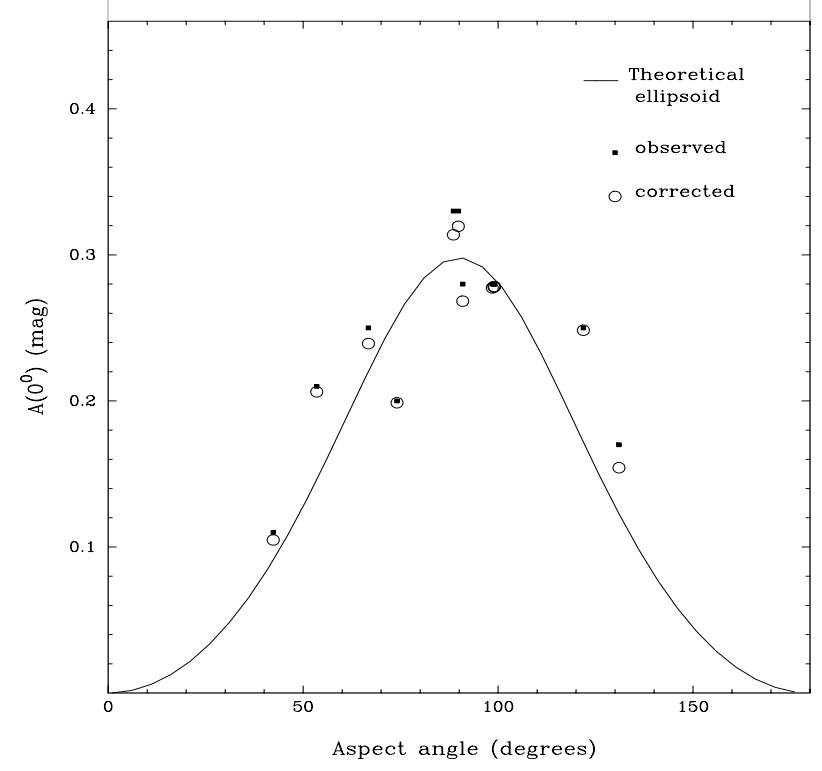

Fig. 2. Amplitude obtained considering 10 Hygiea as a triaxial ellipsoid with $a / b=1.31$ and $b / c=1.2$. Amplitudes observed and corrected by a phase factor $\beta_{\mathrm{A}}=0.001$

$b / c$, and a phase coefficient that takes into account the phase angle effects on the amplitude of the lightcurves, $\beta_{\mathrm{A}}$.

Lightcurve data reported in the literature together with lightcurves obtained here for these asteroids have been used in the analysis. Most of the lightcurves used in this work can be found in the Asteroid Photometric Catalogue by Lagerkvist et al. (1987a, 1988, 1992a).

\section{Results}

\subsection{Hygiea}

10 Hygiea is a C-type asteroid (Tholen 1989) with a diameter of $429 \mathrm{~km}$ (Tedesco 1989). 10 Hygiea has been observed in eight oppositions between 1953 and 1991 (Groeneveld \& Kuiper 1954; Vesely \& Taylor 1985; Lagerkvist et al. 1987b, 1989, 1992c; Michalowski et al. 1991), and now in 1996. 10 Hygiea has been also observed by ISO satellite (Dotto et al. 1998, 1999) and imaged with the Hubble Space Telescope (Storrs et al. 1998, 1999). No companions to 10 Hygiea have been detected down to a limit of seven magnitudes.

From our data we find a synodic period for 10 Hygiea of $27.63 \pm 0.02$ hours. This synodic period is consistent with the sidereal period derived by Michalowski et al. (1991) from all the observations of 10 Hygiea between 1953 and 1989, and also with the observations in 1991 (Erikson \& Magnusson 1993). Figure 1 shows the composite lightcurves of 10 Hygiea derived using this synodic period. Although there is a gap in the coverage of the rotational phase of the lightcurve of this asteroid at the time of one of the minima, the composite lightcurves obtained for this asteroid show two maxima and two minima per rotational cycle in all the uvby filters. The two maxima are of different magnitude by 0 . 06 . The maximum amplitude is of 0.28 in all the uvby filters.

The $b-y$ colour index does not show any variation during the rotational phase of this asteroid (see Fig. 1). The $v-b$ and $u-b$ colour indices seem to show a variation during the rotational phase of this asteroid. These $v-b$ and $u-b$ colour curves seem to be anticorrelated with the uvby lightcurves, although the lack of data that overlap during fractional parts of the rotational phase of this asteroid does not let us to conclude that these detected variations in the $v-b$ and and $u-b$ colour curves are anticorrelated with the uvby lightcurves. Recently, rotational spectral variability of 10 Hygiea, in the $0.7 \mu \mathrm{m}$ region, has been detected by Howell et al. (1999).

The mean values of $B-V=0.66$ and $U-B=0.31$ found for this asteroid, agree with the values, $B-V=$ 0. 69 and $U-B=0.31$, reported by Bowell et al. (1979).

The results obtained here for the sidereal period, pole and shape of 10 Hygiea are Tsid $=1$. 150967 being $\lambda_{\mathrm{p}}=120^{\circ}, \beta_{\mathrm{p}}=34^{\circ}\left(\right.$ or $\left.\lambda_{\mathrm{p}}=295^{\circ}, \beta_{\mathrm{p}}=43^{\circ}\right), a / b=1.31$, $b / c=1.20$ and $\beta_{\mathrm{A}}=0.001$, having a retrograde sense of rotation, in agreement with the later determinations (Michalowski et al. 1991; Michalowski 1993; Erikson \& Magnusson 1993).

The observed amplitudes together with the theoretical amplitudes, at zero-phase angle, obtained with the 


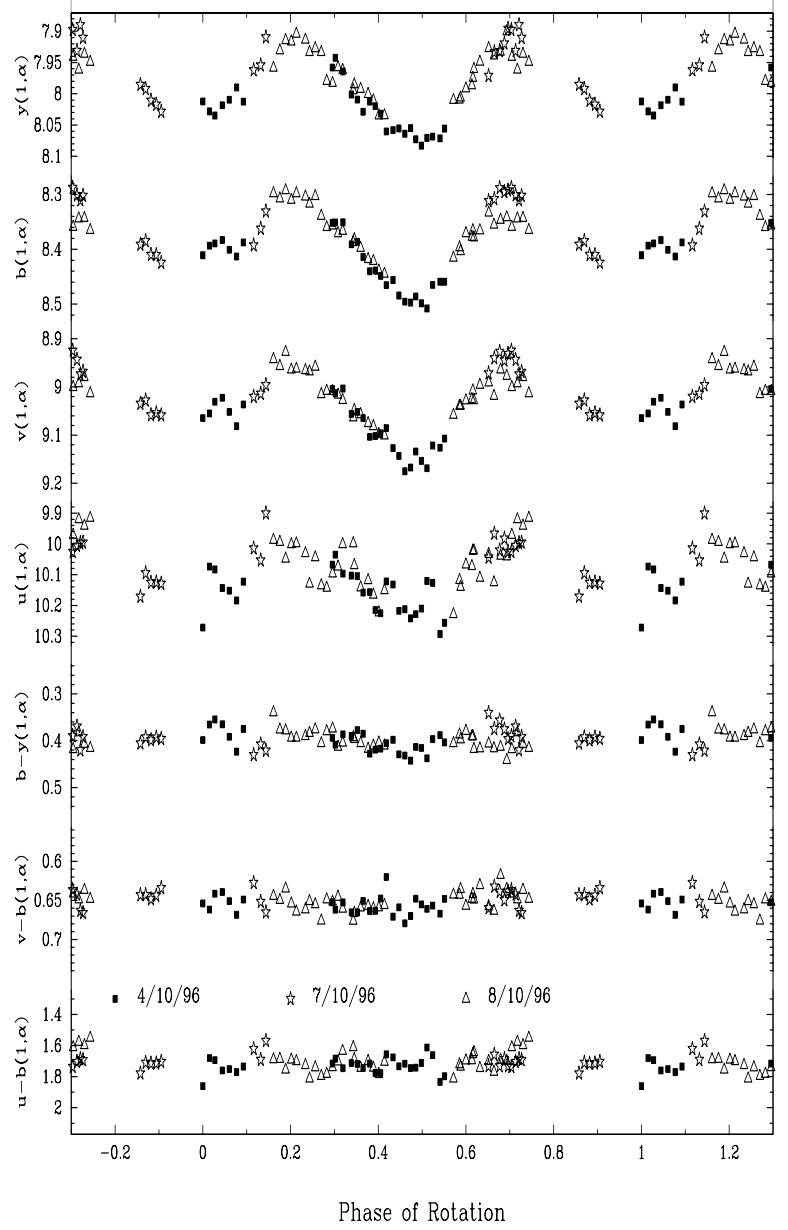

Fig. 3. Lightcurves and colour indices of 241 Germania in rotational phase. The 0 phase time corresponds to JD 2450361.3180 corrected for light-time

solution values of $a / b$ and $b / c$ versus the aspect angle are plotted in Fig. 2.

\subsection{Germania}

241 Germania is a CP-type asteroid (Tholen 1989) with a diameter of $169 \mathrm{~km}$ (Tedesco 1989). This asteroid has been observed in two oppositions in 1990 and 1991 (Lagerkvist et al. 1992b; Shevchenko et al. 1992) and now in 1996. Lagerkvist et al. (1992b) observed 241 Germania during five nights in 1990 and the most likely solution that they found for the synodic period for 241 Germania was about 15.2 hours. Shevchenko et al. (1992) observed 241 Germania during four nights in October-November 1991. They found, for 241 Germania, a synodic period of 8.998 hours from their observations. We find a synodic period of $15.51 \pm 0.01$ hours from our data. This is $18.6 \mathrm{~min}$ utes larger than the one proposed by Lagerkvist et al.

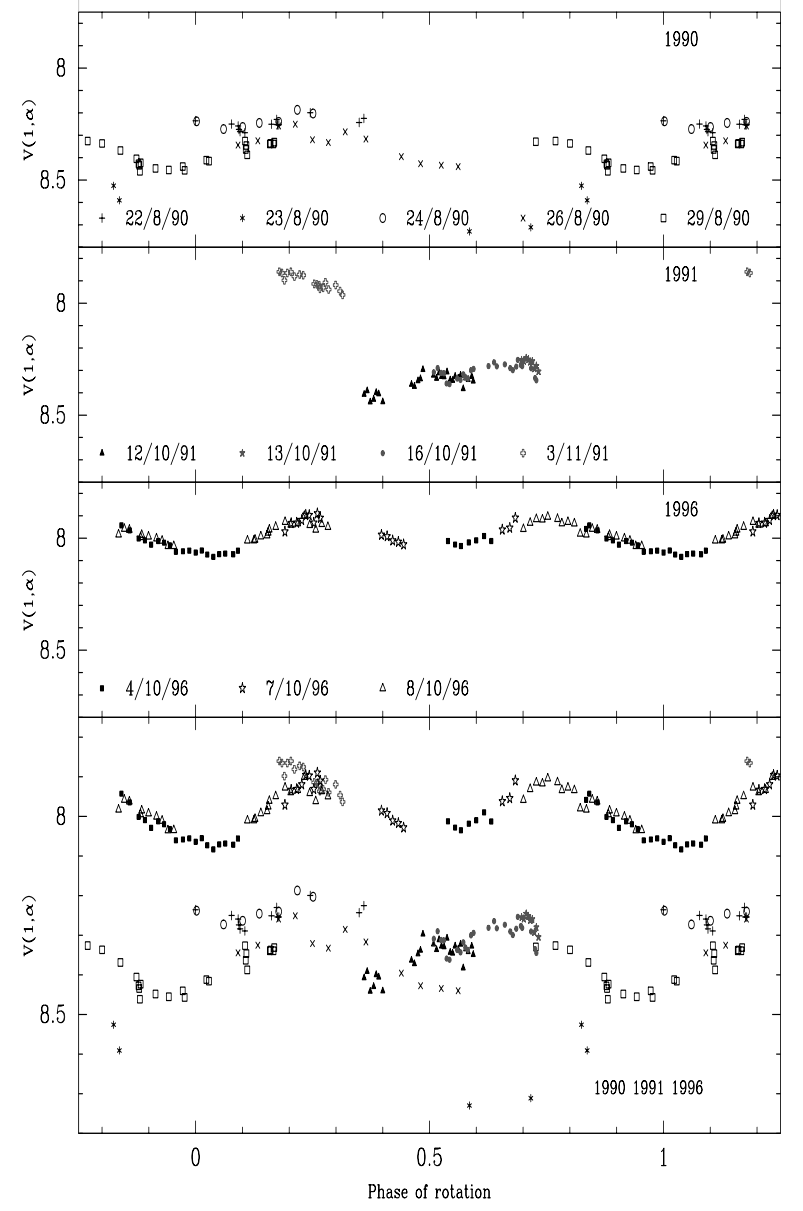

Fig. 4. Lightcurves and colour indices of 241 Germania in rotational phase. The 0 phase time corresponds to JD 2448125.5747 corrected for light-time

(1992b). We tried to make composite lightcurves of our observations using these two previously suggested synodic periods, but none of them were consistent with our observations. The composite lightcurves derived for 241 Germania, from our observations, using a synodic period of 15.51 hours are shown in Fig. 3. We also tried to make composite lightcurves of 1990 and 1991 observations of 241 Germania using the synodic period obtained from our observations. Table 3 contains the date of available observations of 241 Germania together with its ecliptic longitude and latitude, the solar phase angle and the mean reduced magnitude observed, $\bar{V}(1, \alpha)$, during each night. Figure 4 shows the composite lightcurves derived, using a synodic period of 15.51 hours, from 1990, 1991 and 1996 observations. We have not applied any phase correction to the reduced magnitudes, $V(1, \alpha)$, however for the nights $13^{\text {rd }}$ and $16^{\text {th }}$ of October 1991 , where only relative magnitudes are available, we have shifted these relative 
Table 3. Observations of 241 Germania

\begin{tabular}{lrrrr}
\hline $\begin{array}{l}\text { Date } \\
\text { (0 UT) }\end{array}$ & $\begin{array}{r}\text { Long } \\
(1950)\end{array}$ & $\begin{array}{r}\text { Lat } \\
(1950)\end{array}$ & $\begin{array}{r}\text { Phase } \\
(\mathrm{deg})\end{array}$ & $\begin{array}{r}\overline{V(1, \alpha)} \\
(\mathrm{mag})\end{array}$ \\
\hline 241 Germania & & & & \\
22081990 & 301.40 & 5.38 & 9.59 & $8.25 \pm 0.02$ \\
23081990 & 301.27 & 5.39 & 9.93 & $8.64 \pm 0.10$ \\
24081990 & 301.13 & 5.40 & 10.28 & $8.24 \pm 0.03$ \\
26081990 & 300.87 & 5.41 & 10.96 & $8.34 \pm 0.07$ \\
29081990 & 300.52 & 5.42 & 11.95 & $8.39 \pm 0.05$ \\
12101991 & 56.42 & 5.70 & 12.69 & $8.36 \pm 0.04$ \\
13101991 & 56.34 & 5.70 & 12.45 & $-2.23 \pm 0.02$ \\
16101991 & 55.96 & 5.71 & 11.43 & $-4.60 \pm 0.03$ \\
03111991 & 52.96 & 5.59 & 5.01 & $7.90 \pm 0.03$ \\
04101996 & 18.94 & 8.25 & 4.23 & $8.03 \pm 0.04$ \\
07101996 & 18.32 & 8.24 & 3.43 & $7.95 \pm 0.05$ \\
08101996 & 18.11 & 8.23 & 3.23 & $7.96 \pm 0.04$ \\
\hline
\end{tabular}

magnitudes by additional constants of $10{ }^{\mathrm{m}} 5$ and $12^{\mathrm{m}} .9$ for the nights $13^{\text {rd }}$ and $16^{\text {th }}$ of October, respectively. The different value of the solar phase angle on November 3, 1991 (5.01) and on October 12, 1991 (12.69) explains the difference in the magnitudes observed those days. The composite lightcurves obtained from 1990 and 1991 observations seem to be well defined with the data points in their rotational phases. This gives us confidence about the synodic period derived for 241 Germania.

The composite lightcurves obtained here for 241 Germania show two maxima of similar magnitude and two unequal minima per rotational cycle in all the uvby filters. The largest amplitude in all the uvby filters is of 0.17 . The colour indices do not show any significant variation during the rotational phase of this asteroid (see Fig. 3).

The mean values of $B-V=0{ }^{\mathrm{m}} 64$ and $U-B=0.26$ found for this asteroid, are in good agreement with the values $(B-V=0.69$ and $U-B=0.30)$ reported by Bowell et al. (1979).

We have used all the available observations of 241 Germania, from 3 oppositions, to obtain information about its rotational and shape parameters. The most probable solution obtained for the sidereal period, pole and shape is $T$ sid $=0.646605, \lambda_{\mathrm{p}}=94^{\circ}$ and $\beta_{\mathrm{p}}=44^{\circ}$ or $\left(\lambda_{\mathrm{p}}=256^{\circ}\right.$ and $\left.\beta_{\mathrm{p}}=42^{\circ}\right)$ and values for $a / b$ of 1.20 , for $b / c$ of 1.5 and for $\beta_{\mathrm{A}}$ of 0.002 , having a prograde sense of rotation (a retrograde solution with Tsid $=0$ d.647033, might also be possible). No previous solutions have been determined for this asteroid. The prograde solution has slightly smaller residuals. We choose the prograde solution as the most probable.

The observed amplitudes together with the theoretical amplitudes, at zero-phase angle, obtained with these solution values of $a / b$ and $b / c$ versus the aspect angle are plotted in Fig. 5. The agreement is very good, however more lightcurves of 241 Germania would improve

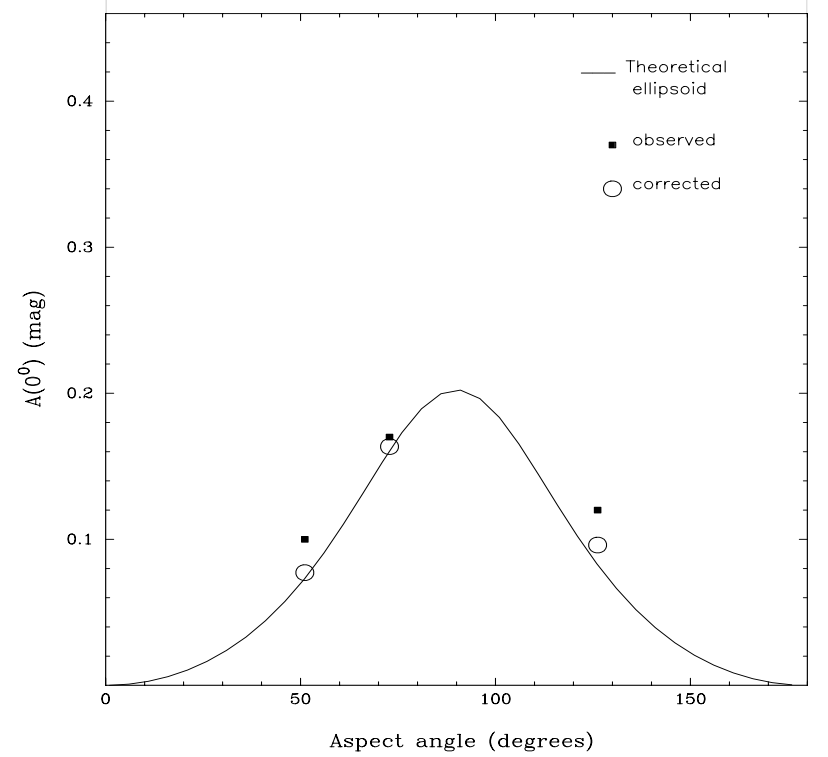

Fig. 5. Amplitude obtained considering 241 Germania as a triaxial ellipsoid with $a / b=1.2$ and $b / c=1.5$ Amplitudes observed and corrected by a phase factor $\beta_{\mathrm{A}}=0.002$

the determination of its rotational and shape parameters. According to the poles presented here, during the next oppositions in May/June $2000\left(\lambda=245^{\circ}\right)$ and in December $2002\left(\lambda=80^{\circ}\right)$, the lightcurves of 241 Germania will have small amplitudes, while during the oppositions in September $2001\left(\lambda=342^{0}\right)$ and in the February/March $2004\left(\lambda=150^{\circ}\right)$, the aspect of 241 Germania will be close to equatorial, and then, the lightcurves will have larger amplitudes. Those observations would help to improve the results presented here.

\subsection{Iolanda}

This object is of S-type (Tholen 1989) with a diameter of $59 \mathrm{~km}$ (Tedesco 1989). There are no available lightcurves for this asteroid in previous oppositions.

We find a synodic period for 509 Iolanda of $12.72 \pm$ 0.02 hours. The composite lightcurves obtained for 509 Iolanda show two maxima and two minima per rotational cycle (see Fig. 6). There are gaps in the coverage of the lightcurve of this asteroid, and only one maximum and minimum are completely defined. The largest amplitude observed in all the uvby filters is greater than $0{ }^{\mathrm{m}} 45$.

The colour indices obtained for this asteroid show large dispersions and no significant variations are detected in the colour indices along the rotational phase. We find a mean value of $U-B=0.43$ colour index that agrees with the value, $U-B=0$. 41 , reported by Bowell et al. (1979). Although the mean value of $B-V=0.70$ obtained is smaller than the value, $B-V=0$ m 83 , reported by Bowell et al. (1979), the difference between the value obtained 


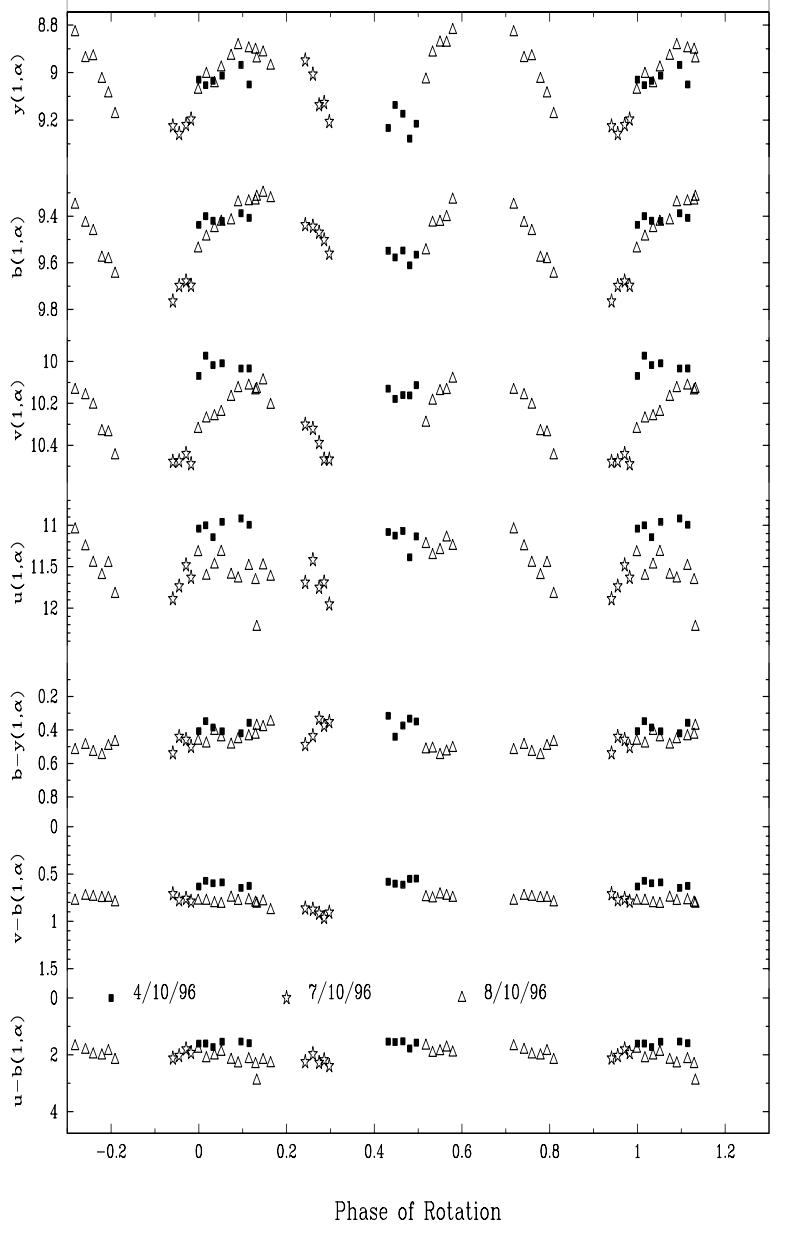

Fig. 6. Lightcurves and colour indices of 509 Iolanda in rotational phase. The 0 phase time corresponds to JD 2450361.3211 corrected for light-time

and the one reported is within the large error bars of the determination and thus may not be significant.

There are not enough lightcurves to deduce the sidereal period, pole and shape of 509 Iolanda. For this asteroid, lightcurves taken at different ecliptic longitudes are needed to determine its rotational and shape parameters.

\section{Conclusions}

Lightcurves of asteroids 10 Hygiea, 241 Germania and 509 Iolanda, obtained during September-October 1996 using uvby Strömgren photometry, are presented. The lightcurves obtained for each of these three asteroids show two maxima and two minima per cycle. No significant tendencies are found in the colour indices of 241 Germania and 509 Iolanda during their rotational phases. However, a slight variation is detected in the the $v-b$ and $u-b$ colour indices of 10 Hygiea during its rotational phase. The values for the sidereal period, the pole and the shape parameters derived for 10 Hygiea are in agreement with previous results. A first estimation for the sidereal period, the pole position and shape of 241 Germania is presented and a value for the synodic period of 509 Iolanda is proposed.

Acknowledgements. This research was partially supported by the Comisión Interministerial de Ciencia y Tecnología under contracts ESP97-1773-C03-01 and ESP97-1798, the Junta de Andalucía and the Dirección General de Enseñanza Superior e Investigación Científica (DGESIC) under project PB98-0499. This research has made use of the Asteroid Photometric Catalogue database. We very gratefully acknowledge the staff of Sierra Nevada Observatory for their help during the run of observations. Acknowledgements are also especially made to M.C. Romero for making available many papers used in this investigation and to V.G. Brown for proofreading. We wish to thank the referee, A. Harris, for his useful comments and suggestions.

\section{References}

Bowell E., Gehrels T., Zellner B., 1979, in Asteroids I, Gehrels T. (ed.). Univ. of Arizona press, Tucson, p. 1108

De Angelis G., 1993, PSS 41, 285

Dotto E., Barucci M.A., Crovisier J., et al., 1998, BAAS, DPS $30,5.01$

Dotto E., Barucci M.A., Müller T., et al., 1999, BAAS, DPS $31,4.04$

Erikson A., Magnusson P., 1993, Icarus 103, 62

Groeneveld I., Kuiper G.P., 1954, ApJ 120, 529

Hauck B., Mermilliod M., 1998, A\&AS 129, 431

Howell E.S., Rivkin A.S., Soderberg A., Vilas F., Cochran A.L., 1999, BAAS, DPS 31, 4.01

Lagerkvist C.-I., Barucci M.A., Capria M.T., et al., 1987a, Asteroid Photometric Catalogue, Consiglio Nazionale delle Richerche, Roma

Lagerkvist C.-I., Hahn G., Magnusson P., Rickman H., 1987b, A\&AS 70, 21

Lagerkvist C.-I., Barucci M.A., Capria M.T., et al., 1988, Asteroid Photometric Catalogue, First Update, Consiglio Nazionale delle Richerche, Roma

Lagerkvist C.-I., Magnusson P., Williams I.P., et al., 1989, A\&AS 95, 461

Lagerkvist C.-I., Barucci M.A., Capria M.T., et al., 1992a, Asteroid Photometric Catalogue, Second Update, Uppsala Universitet, Uppsala

Lagerkvist C.-I., Magnusson P., Debehogne H., et al., 1992b, A\&AS 95, 461

Lagerkvist C.-I., Oja T., Pohjolainen S., Erikson A., 1992c, MPB 19, 29

López-González M.J., Rodríguez E., 1999, A\&AS 139, 565

Magnusson P., 1986, Icarus 68, 1

Magnusson P., Barucci M.A., Drummond J.D., et al., 1989, in Asteroids II, Binzel R.P., Gehrels T. and Matthews M.S. (eds.), p. 66

Michalowski T., 1993, Icarus 106, 563

Michalowski T., Velichko F.P., 1990, Acta Astron. 40, 321 
Michalowski T., Velichko F.P., Lindgren M., et al., 1991, A\&AS 91,53

Nielsen R.F., 1983, Inst. Theor. Astrophys. Oslo Report No. 59, Hauge O. (ed.), p. 141

Rodríguez E., González-Bedolla S.F., Rolland A., Costa V., López de Coca P., 1997, A\&A 324, 959

Rodríguez E., Rolland A., López-González M.J., Costa V., 1998, A\&A 338, 905

Shevchenko V.G., Chiornij V.G., Krugly Yu N., et al., 1992, Icarus 100, 295

Storrs A., Wells E., Zellner B., Stern A., Durda D., 1998,
BAAS, DPS 30, 7.12

Storrs A., Wells E., Stern A., Zellner B., 1999, BAAS, DPS 31, 11.03

Taylor R.C., 1979, in Asteroids I, Gehrels T. (ed.). Univ. of Arizona press, Tucson, p. 480

Tedesco E.F., 1989, in Asteroids II, Binzel R.P., Gehrels T. and Matthews M.S. (eds.), p. 1090

Tholen D.J., 1989, in Asteroids II, Binzel R.P., Gehrels T. and Matthews M.S. (eds.), p. 1139

Vesely C.D., Taylor R.C., 1985, Icarus 64, 37

Warren W.H., Hesser J.E., 1977, ApJS 34, 207 